\title{
Engineering of radiation of optically active molecules with chiral nano-meta-particles
}

\author{
Vasily V. Klimov ${ }^{1 *}$, Dmitry V. Guzatov ${ }^{2}$, Martial Ducloy ${ }^{3}$ \\ ${ }^{1}$ P.N. Lebedev Physical Institute, Russian Academy of Sciences, 53 Leninsky Prospect, 119991 Moscow, Russia \\ ${ }^{2}$ Yanka Kupala Grodno State University, 22 Ozheshko str., 230023 Grodno, Belarus and \\ Laboratoire de Physique des Lasers, UMR CNRS 7538, Institut Galilee, \\ Universite Paris-Nord, Avenue J-B. Clement, F 93430 Villetaneuse, France
}

(Dated: May 19, 2018)

The radiation of optically active (chiral) molecule placed near chiral nanosphere is investigated. The optimal conditions for engineering of radiation of optically active (chiral) molecules with the help of chiral nanoparticles are derived. It is shown that for this purpose, the substance of the chiral particle must have both $\varepsilon$ and $\mu$ negative (double negative material (DNG)) or negative $\mu$ and positive $\varepsilon$ ( $\mu$ negative material (MNG)). Our results pave the way to an effective engineering of radiation of "left" and "right" molecules and to creating pure optical devices for separation of drugs enantiomers.

It is well known that nanoparticles influence substantially both fluorescence of molecules and Raman scattering of light by molecules. These effects are especially strong in the case of metallic nanoparticles where plasmon resonances can be excited. As a result, it is possible to detect radiation even from single molecule (surface enhanced Raman scattering SERS [1] and surface enhanced fluorescence SEF [2]) and to use this effect in various applications, in particular, for detection of certain proteins in disease diagnosis [3]. Recently it was shown that planar chiral metamaterials [4] and even nonchiral plasmonic nanoparticles [5] are able to dramatically (by several orders of magnitude) change the chiral dichroism of a chiral molecule and thus such metamaterials could form the basis for assaying technologies capable of detecting amyloid diseases and certain types of viruses.

Interesting effects are also found in the study of influence of chiral nanoparticles [6] and nanoparticles with negative refraction index [7] on electric dipole radiations of atoms and molecules. Full quantum-electrodynamics theory of influence of nanospheres of various composition including media with negative refraction index on radiation of electric and magnetic dipoles was developed in [8]. However, the interference effects between these dipoles were not investigated there.

Even more interesting problem is to investigate the possibility of controlling radiation of optically active molecules [9]. As far as we know this problem has not been investigated until now. The goal of present work is to investigate the influence of chiral nanoparticles on spontaneous emission of optically active molecules and to show that it is possible to discriminate drug enantiomers with pure optical methods. Geometry of the problem is shown in Fig. 1.

As the model of an optically active molecule we assume

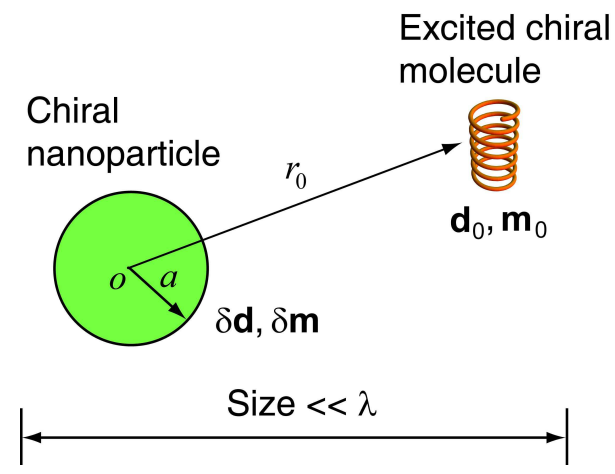

FIG. 1: Geometry of the considered problem. An optically active (chiral) excited molecule is placed near chiral spherical nanoparticle having radius $a$. The total size of the region of molecule and nanobody is much less than the wavelength $\lambda$. Molecule is placed at the distance $r_{0}$ from the center of co-ordinates, which coincides with the nanoparticle center, and characterized by its electric $\left(\mathbf{d}_{0}\right)$ and magnetic $\left(\mathbf{m}_{0}\right)$ dipole moments which induce corresponding dipole moments $\delta \mathbf{d}$ and $\delta \mathbf{m}$ of nanoparticle.

that its radiation is of both electric dipole and magnetic dipole nature, i.e. that the Hamiltonian of molecule interaction with field has the form [10]

$$
\hat{H}_{i n t}=-\hat{\mathbf{d}} \mathbf{E}-\hat{\mathbf{m}} \mathbf{H} .
$$

Matrix elements of operators will be written as $\mathbf{d}_{0}$ and $-i \mathbf{m}_{0}=-i \xi \mathbf{d}_{0}$ due to the fact that the magnetic moment operator is purely imaginary. Parameter $\xi$ characterize relative value of magnetic dipole moment. Within this model, we will refer to a molecule for which $\mathbf{d}_{0}$ and $\mathbf{m}_{0}$ are parallel as a "right" molecule, whereas the molecule in which the magnetic moment is antiparallel to the electric dipole moment will be referred to as a "left" molecule. Thus, we are considering a molecule in which the electrons are constrained to move along a helical path. This picture also explains the choice of the phase difference between electric and magnetic dipole moments.

To control the radiation of molecule with a particular chirality it is necessary to create such nanoenvironment that it forces interference between electric and magnetic dipole radiation of molecules. Such fields can be created with the help of chiral nanoparticles, i.e. nanoparticles, which can be described with Drude-Born-Fedorov constitutive relations [11]: 


$$
\begin{aligned}
& \mathbf{D}=\varepsilon(\mathbf{E}+\beta \nabla \times \mathbf{E}) ; \\
& \mathbf{B}=\mu(\mathbf{H}+\beta \nabla \times \mathbf{H})
\end{aligned}
$$

where $\mathbf{D}, \mathbf{E}$ and $\mathbf{B}, \mathbf{H}$ are the inductions and the strengths of electric and magnetic fields, respectively, $\varepsilon$, $\mu$ are the dielectric permittivity and the magnetic permeability of chiral media, $\beta$ is the dimensional chirality parameter. The dimensionless chirality parameter $\chi$ is defined by relation $\chi=\beta k_{0}$, where $k_{0}=\omega / c$ is the wavenumber in vacuum.

The simplest example of chiral nanoparticles is a drop of sugar solution. Today, thanks to the development of nanotechnology it is possible to prepare chiral nanoparticles of various forms. As an example, one should note the artificially prepared chiral nanoparticles in the form of segments of nanospirals [12] or from asymmetric clusters of spherical nanoparticles [13]. An important way to obtain chiral nanoparticles is the introduction of metal nanoparticles into spiral organic molecules [14]. In the present work for simplicity we will consider a homogeneous spherical nanoparticle, the substance of which is described by constitutive equations (2). The problem of scattering of plane waves of different polarizations on chiral spherical particle can be solved exactly by analogy with the Mie solution [15, 16]. In Fig. 2 the structure of TM plasmonic resonances for spherical nanoparticle of radius $a$ without chirality and with chirality factor $\chi=\beta k_{0}=0.1$, constructed within the framework of solutions [15, 16] is shown.

From this figure it is seen, that TM modes in nonchiral nanoparticle have electric dipole plasmon resonance at $\varepsilon \approx-2$, nearly independent of magnetic permeability. Any chirality - even small - of the nanoparticle (see Fig. (2b) leads to a radical change in the shape of the resonance line, which becomes dependent in a non-trivial way on both the dielectric and the magnetic permeability of the material from which the nanoparticle is made. Below we will see that these "hybrid" chiral-plasmon modes in nanoparticle provide the effective interference between radiation of electric and magnetic dipole moments of chiral molecule.

To estimate the interaction of the radiating optically active molecule with chiral nanoparticle we can neglect retardation effects (see Fig. 11). In this case, in the field of radiating chiral molecules the near-field is dominating

$$
\begin{aligned}
\mathbf{E}_{0} & =\frac{3 \mathbf{n}\left(\mathbf{n d}_{0}\right)-\mathbf{d}_{0}}{r^{3}} ; \\
\mathbf{H}_{0} & =-i \frac{3 \mathbf{n}\left(\mathbf{n m}_{0}\right)-\mathbf{m}_{0}}{r^{3}}
\end{aligned}
$$

where $r$ is the distance between the molecule and the observation point, $\mathbf{n}$ is the unit vector in the direction from the molecule to the observation point, and the monochromatic time dependence of fields $\left(e^{-i \omega t}\right)$ is omitted.

Fields (3) induce the electric $\delta \mathbf{d}$ and the magnetic $\delta \mathbf{m}$ dipole moments in nanoparticle:
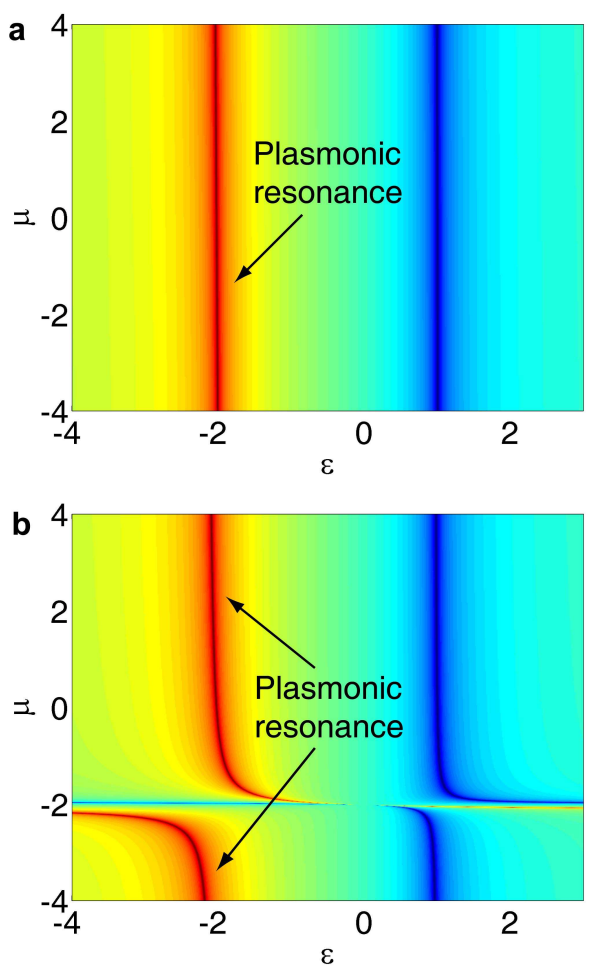

FIG. 2: Structure of TM resonances of spherical nanoparticle. Dependence of one of the Mie's coefficients $(n=1)$ for spherical particle [16] as the function of dielectric permittivity $(\varepsilon)$ and magnetic permeability $(\mu)$ is shown. Permittivity and permeability of the medium in which nanoparticle is situated are equal to unit. a, Nanoparticle without chirality. b, Nanoparticle with nonzero chirality $\chi=\beta k_{0}=0.1$. Size parameter of nanosphere is $k_{0} a=0.1$, where $k_{0}$ is the wavenumber in vacuum.

$$
\begin{aligned}
\delta \mathbf{d} & =\alpha_{E E} \mathbf{E}_{0}\left(\mathbf{r}_{0}\right)+\alpha_{E H} \mathbf{H}_{0}\left(\mathbf{r}_{0}\right) \\
\delta \mathbf{m} & =\alpha_{H E} \mathbf{E}_{0}\left(\mathbf{r}_{0}\right)+\alpha_{H H} \mathbf{H}_{0}\left(\mathbf{r}_{0}\right)
\end{aligned}
$$

where $\alpha_{E E}, \alpha_{E H}, \alpha_{H E}$ and $\alpha_{H H}$ are the tensors of electromagnetic polarizability of spherical nanoparticle in uniform external fields and $\mathbf{r}_{0}$ is the radius-vector of the molecule's position. The possibility of applying the formula (4) to strongly nonuniform fields (3) is associated with an unique property [17] of ellipsoids and spheres, according to which the tensor of polarizability of an ellipsoid in nonuniform field of dipole source is defined by usual polarizabilities and by dipole field, which is averaged over the volume of particle. In the case of spheres such an averaging gives value of the dipole field in the center of sphere, i.e. $\mathbf{E}_{0}\left(\mathbf{r}_{0}\right)$ and $\mathbf{H}_{0}\left(\mathbf{r}_{0}\right)$, respectively.

For a spherical particle with constitutive relations (2) it is easily to find that the quasistatic electromagnetic polarizabilities are 


$$
\begin{aligned}
\alpha_{E E} & =a^{3} \frac{(\varepsilon-1)(\mu+2)+2 \varepsilon \mu \chi^{2}}{(\varepsilon+2)(\mu+2)-4 \varepsilon \mu \chi^{2}} ; \\
\alpha_{H H} & =a^{3} \frac{(\mu-1)(\varepsilon+2)+2 \varepsilon \mu \chi^{2}}{(\varepsilon+2)(\mu+2)-4 \varepsilon \mu \chi^{2}} ; \\
\alpha_{E H}=-\alpha_{H E} & =a^{3} \frac{3 \chi \varepsilon \mu i}{(\varepsilon+2)(\mu+2)-4 \varepsilon \mu \chi^{2}}
\end{aligned}
$$

where $a$ is nanosphere radius, $\chi=\beta k_{0}, \varepsilon$ and $\mu$ are dimensionless chirality parameter, dielectric permittivity and magnetic permeability of the nanoparticle, respectively, and it is assumed that the particle is located in vacuum.

Since the total size of the molecule and nanoparticle system is much less than the wavelength, the dipole momenta of molecule and particle are added in phase and the decay rate of radiation from such a composite system is described by

$$
\Gamma_{\text {rad }}=\frac{4 k_{0}^{3}}{3 \hbar}\left|\mathbf{d}_{0}+\delta \mathbf{d}\right|^{2}+\frac{4 k_{0}^{3}}{3 \hbar}\left|\mathbf{m}_{0}+i \delta \mathbf{m}\right|^{2}
$$

where $\hbar$ is the Planck's constant.

Since the orientation of a molecule can be arbitrary relative to the particle, to obtain an effective decay rate one should averaged (6) over the orientations of the molecule, or, equivalently, over the unit vector $\mathbf{n}$. As a result we obtain

$$
\begin{aligned}
\Gamma_{\text {rad,eff }}= & \frac{4 k_{0}^{3}\left|\mathbf{d}_{0}\right|^{2}}{3 \hbar}\left(1+\frac{2}{r_{0}^{6}}\left|\alpha_{E E}-i \xi \alpha_{E H}\right|^{2}\right. \\
& \left.+|\xi|^{2}+\frac{2}{r_{0}^{6}}\left|i \alpha_{H E}+\xi \alpha_{H H}\right|^{2}\right)
\end{aligned}
$$

where $\xi$ defined by relation $\mathbf{m}_{0}=\xi \mathbf{d}_{0}$.

Usually, the electric dipole moment of the molecule is much larger than the magnetic dipole moment $\left|\mathbf{d}_{0}\right| \gg$ $\left|\mathbf{m}_{0}\right|$ or $\xi \ll 1$. Chirality parameter, even in the hypothetical metamaterials is also small $\chi \ll 1$. Because of this, the first (electric) term in (77) is usually greater than the second (magnetic) one. Therefore, the interference between electric and magnetic fields is possible only when two non-trivial conditions take place.

1. The system must have chiral-plasmon resonance, that is, the following condition should be satisfied

$$
(\varepsilon+2)(\mu+2)-4 \varepsilon \mu \chi^{2}=0
$$

(cf. Fig. 2a). Under this condition, the contribution of magnetic radiation increases.

2. The electric dipole moment induced in the nanoparticle must be zero, that is the following condition should take place

$$
\alpha_{E E}-i \xi \alpha_{E H}=0
$$

The simultaneous solution of (8) and (9) determines the values of dielectric permittivity and magnetic permeability of nanoparticle where the radiative decay rate of chiral molecules is minimal and, therefore, the interference between electric dipole and magnetic dipole radiations is maximal

$$
\begin{aligned}
& \mu * \rightarrow-\frac{2 d_{0}}{d_{0}+2 m_{0} \chi} \\
& \varepsilon * \rightarrow-\frac{2 m_{0}}{m_{0}+2 d_{0} \chi} .
\end{aligned}
$$

Equations (10) are the key equations for chiral molecule discrimination and enantiomer selectivity.

It is very important to note that when the sign of $m_{0}$ is changed, i.e. when changing the chirality of the molecule the "resonance" magnetic permeability varies slightly and remains approximately equal to -2 . On the other hand, the "resonance" permittivity has different signs for the molecules of different chirality. This means that both nanoparticles with simultaneously negative $\varepsilon$ and $\mu$ (DNG metamaterials), and nanoparticles with negative $\mu$ and positive $\varepsilon$ (MNG metamaterials or magnetic plasma) are suitable for the effective control of the radiation of chiral molecules. From point of view of practical implementation of such control the MNG nanoparticles seem to be the most suitable, because they can be realized within well-developed technology of Split Rings Resonators [18, 19].

Fig. [3] shows the decay rates of the "right" (Fig. 22a) and "left" (Fig. 20) molecules on the dielectric permittivity and magnetic permeability of the material from which the nanoparticle is made.

This figure shows that, indeed, for the values of dielectric permittivity and magnetic permeability, as determined by the equation (10), the decay rates of "right" (Fig. 3b) or "left" (Fig. 3b) molecules are close to zero. It is this fact that determines the possibility of their separation.

To determine a quantitative measure of discrimination, the ratio of the decay rate of the "left" molecules to the decay rate of the "right" ones and vice versa is shown in Fig. [4.

This figure shows that, indeed, for the chosen parameters, there is a difference in the radiative decay rates of "right" and "left" molecules up to 10 or 50 or more times depending on the chirality of molecule considered as a reference one. In other words, the nanoparticles with parameters (10) will enhance radiation of the "right" molecules and slow down radiation of the "left" molecules, and vice versa. Let us stress that "lefthandedness" of chiral sphere (DNG material) or its negative $\mu$ (MNG material) are of crucial importance for such discrimination.

Until now we have considered the usual case when the electric dipole momentum of molecule is greater than the magnetic dipole momentum, that is the case when $\xi \ll 1$. In hypothetic case when the magnetic dipole momentum 

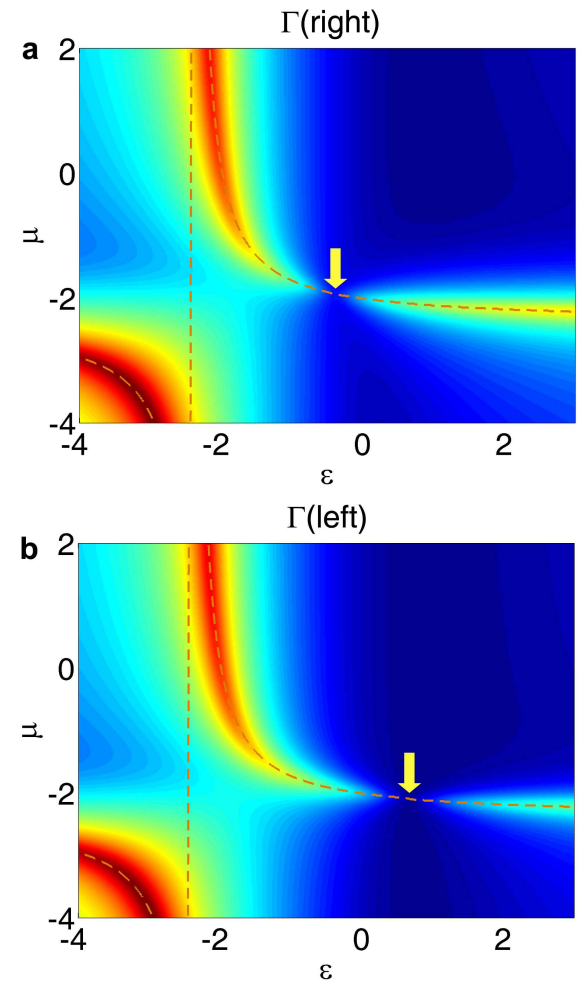

FIG. 3: Effective radiative decay rate of chiral molecule placed near chiral nanoparticle. Effective radiative decay rate (see equation (17) ) is shown as the function of $\varepsilon$ and $\mu$. a, The case of "right" molecule with $m_{0} / d_{0}=\xi=+0.1$. b. The case of "left" molecule with $m_{0} / d_{0}=\xi=-0.1$. Spherical nanoparticle is made from material with chirality $\chi=0.2$; imaginary parts of dielectric permittivity and magnetic permeability are $\varepsilon^{\prime \prime}=\mu^{\prime \prime}=0.1$. The dashed line corresponds to position of the chiral-plasmon resonance (8). Yellow arrow indicates the minimum rates on the resonance curve. Molecule is placed close to nanoparticle surface.

is dominating, that is, in the case where $\xi \gg 1$ it follows from equation (9) that one needs making use of DNG or $\varepsilon$ negative (ENG) materials to obtain enhancement of radiation of "right" or "left" molecule.

The proposed theory allows one to estimate the parameters of nanoparticles required for the discrimination of the radiation of "left" or "right" molecules. It is very important that, despite the simple character of the proposed theory, it is accurate enough. The accuracy of the present approach directly follows from the comparison with the results of full quantum-electrodynamics calculations performed by us without any approximations [20].

The predicted effect of influence of chiral nanoparticles on the radiation of optically active molecules can be used in many applications. Here we mention only two, the most obvious ones. The first application is observing separately "right" or "left" molecules with a scanning microscope (see Fig. 5).

To do so, specially tuned (see equation (10) nanopar- a

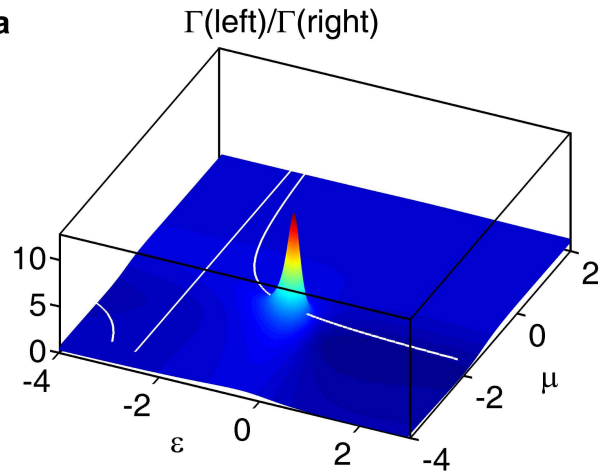

b

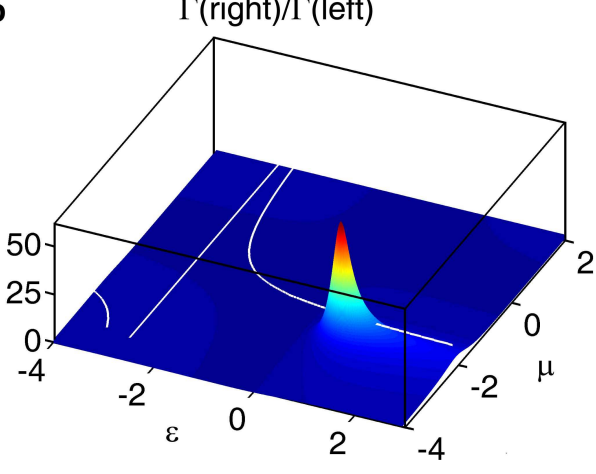

FIG. 4: Relation between effective radiative decay rates of molecules with different chirality. a, The ratio of effective radiative decay rate (see equation (77) of the "left" molecule with $\xi=-0.1$ to the radiative decay rate of the "right" molecule with $\xi=+0.1$. b, The ratio of effective radiative decay rate (see equation (7)) of the "right" molecule with $\xi=+0.1$ to the radiative decay rate of the "left" molecule with $\xi=-0.1$. The dependence on $\varepsilon$ and $\mu$ is presented. Spherical nanoparticle is made from material with chirality $\chi=0.2$, imaginary parts of dielectric permittivity and magnetic permeability are $\varepsilon^{\prime \prime}=\mu^{\prime \prime}=0.1$. The white line shows the position of chiral-plasmon resonance of nanoparticles (see equation (8) and Fig. 3). Molecule is placed close to nanoparticle surface.

ticles are attached to the tip of a scanning microscope to increase the spontaneous emission of a molecule with a selected chirality. A short metallic spiral can be used as such nanoparticle, because it can have required chirality and negative magnetic response. Indeed, the intensity of the fluorescence of a molecule is defined by relation

$$
I_{\text {fluor }}=\hbar \omega \frac{\Gamma_{\text {pump }} \Gamma_{\text {rad }}}{\Gamma_{\text {pump }}+\Gamma_{\text {nonrad }}+\Gamma_{\text {rad }}}
$$

where $\Gamma_{\text {pump }}, \Gamma_{\text {rad }}$ and $\Gamma_{\text {nonrad }}$ are the rate of excitation, radiative and nonradiative decays of a molecule, respectively. In the presence of nanoparticles with the parameters (10) the radiative decay rate of one sort of molecules (e.g. "right" ones) increases, while the radiative decay rate of molecules with opposite chirality ("left") is inhibited. Due to this fact, the contrast between the bright- 


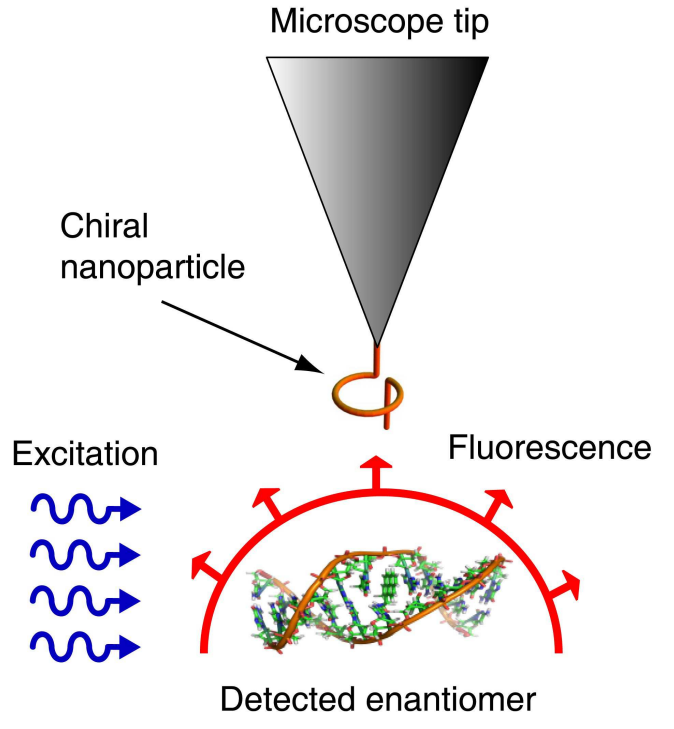

FIG. 5: Operation principle of the nanoscope to distinguish "right" and "left" enantiomers. Chiral nanoparticle (or short nanospiral) is placed on the tip of the microscope. Such nanoparticle is specially tuned to satisfy conditions (10). In such a way miscroscope is tuned to accelerate the radiation of a molecule with selected ("left" or "right") chirality depending on the chirality of the nanoparticle. Excitated molecule with unknown chirality is radiating and its radiation is detected (or not detected) by the microscope, depending on the chirality of molecule. ness of the "right" and the "left" molecules can reach value of 10-100 or more times and, consequently, one will see images of molecules with chosen chirality only.

Significantly more important application of our results is the separation of enantiomers in racemic mixtures. The operation principle of such a device is shown in Fig. 6.

The key element in this scheme is the reaction chamber, which contains nanoparticles prepared in accordance with equation (10). The racemic mixture of enantiomers is placed in this chamber and then excited by one or another way (e.g. photoexcitation). Due to presence of chiral nanoparticles one type of the optically active enantiomers radiates efficiently and goes to ground state quickly, while the remaining excited enantiomers can be ionized by a resonant field, and then removed from the chamber. Other methods of removal of excited molecules or their decay products are also possible. As a result, the desired pure enantiomer will be accumulated in the chamber. Our approach for pure optical spatial separation of enantiomers has some advantages in comparison with other proposal [21], because we have no need to use very low temperatures.

In conclusion, the theory of radiation of optically active molecule placed near chiral nanoparticles is elaborated. It is shown that for special parametres of nanoparticle (DNG, MNG or ENG metamaterials) it is possible to increase substantially radiation of "right" molecules and suppress radiation of "left" molecules and vice versa. An application of this effect to separate racemic mixtures of drug enantiomers is proposed.
[1] Kneipp, K., Moskovits, M. \& Kneipp, H. (eds) SurfaceEnhanced Raman Scattering (Springer-Verlag, Berlin, 2006).

[2] Lakowicz, J. R., Malicka, J., Gryczynski, I., Gryczynski, Z. \& Geddes, C. D. Radiative decay engineering: the role of photonic mode density in biotechnology. J. Phys. D 36, 240-249 (2003).

[3] Manno, D., Filippo, E., Fiore, R., Serra, A., Urso, E., Rizzello, A. \& Maffia, M. Monitoring prion protein expression in complex biological samples by SERS for diagnostic applications. Nanotechnology 21, 165502 (2010).

[4] Hendry, E., Carpy, T., Johnston, J., Popland, M., Mikhaylovskiy, R. V., Lapthorn, A. J., Kelly, S. M., Barron, L. D., Gadegaard, N. \& Kadodwala, M. Ultrasensitive detection and characterization of biomolecules using superchiral fields. Nature Nanotech. 5, 783-787 (2010).

[5] Govorov, A. O., Fan, Z., Hernandez, P., Slocik, J. M. \& Naik, R. R. Theory of circular dichroism of nanomaterials comprising chiral molecules and nanocrystals: Plasmon enhancement, dipole interactions, and dielectric effects. Nano Lett. 10, 1374-1382 (2010).

[6] Lakhtakia, A., Varadan, V. K. \& Varadan, V. V. Radiation by a point electric dipole embedded in a chiral sphere. J. Phys. D 23, 481-485 (1990).

[7] Klimov, V. V. Spontaneous emission of an excited atom placed near a "left-handed" sphere. Opt. Commun. 211, 183-196 (2002).

[8] Klimov, V. V. \& Letokhov, V. S. Electric and magnetic dipole transitions of an atom in the presence of spherical dielectric interface. Laser Phys. 15, 61-73 (2005).

[9] Barron, L. D. Molecular Light Scattering and Optical Activity (Cambridge University Press, Cambridge, 1982).

[10] Schellman, J. A. Circular dichroism and optical rotation. Chem. Rev. 75, 323-331 (1975).

[11] Bokut, B. V., Serdyukov, A. N. \& Fedorov, F. I. On the phenomenological theory of optically active crystals. Kristallografiya 15, 1002-1006 (1970) [Sov. Phys. Crystallogr. 15, 871-875 (1971)].

[12] Gansel, J. K., Thiel, M., Rill, M. S., Decker, M., Bade, K., Saile, V., von Freymann, G., Linden, S. \& Wegener, M. Gold helix photonic metamaterial as broadband circular polarizer. Science 325, 1513-1515 (2009).

[13] Fan, Z. \& Govorov, A. O. Plasmonic circular dichroism of chiral metal nanoparticle assemblies. Nano Lett. 10, 2580-2587 (2010).

[14] Oh, H. S., Liu, S., Jee, H. S., Baev, A., Swihart, M. T. \& Prasad, P. N. Chiral poly(fluorene-alt-benzothiadiazole) (PFBT) and nanocomposites with gold nanoparticles: plasmonically an structurally enhanced chirality. J. Am. Chem. Soc. 132, 17346-17348 (2010). 


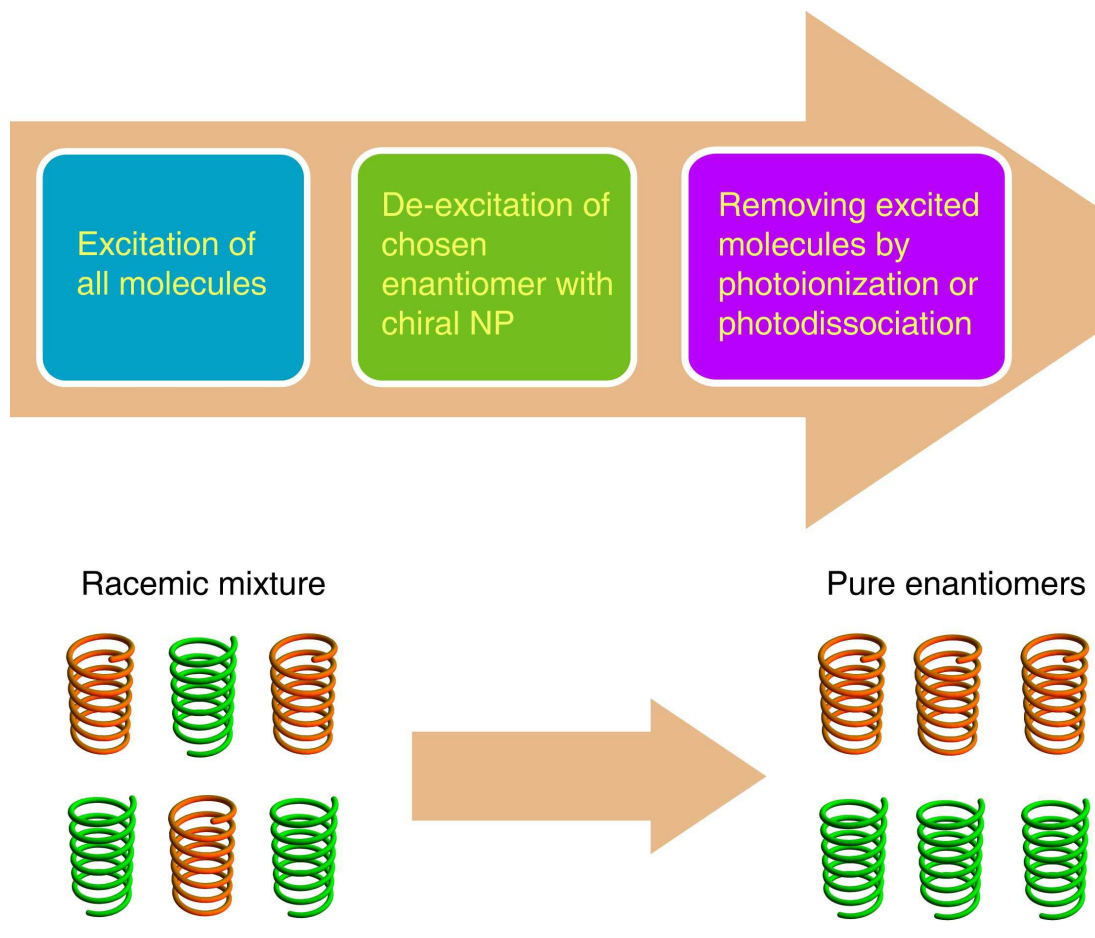

FIG. 6: Operation principle for separating of "right" and "left" enantiomers. The racemic mixture with excited enantiomers of both types of chirality is placed in the specially created reaction chamber. Effective interaction of the chiral nanoparticle which is specially tuned (according to conditions (10) takes place only with molecules with selected chirality. It allows them quickly became unexcited, whereas molecules with another type of chirality remaining excited. Such molecules can be removed from the chamber by different methods. As a result, the desired pure enantiomer will be accumulated in the chamber.

[15] Bohren, C. F. Light scattering by an optically active sphere. Chem. Phys. Lett. 29, 458-462 (1974)

[16] Bohren, C. F. \& Huffman, D. R. Absorption and Scattering of Light by Small Particles (John Wiley-Interscience, New York, 1983).

[17] Guzatov, D. V. \& Klimov, V. V. Radiative decay engineering by triaxial nanoellipsoid. Chem. Phys. Lett. 412, 341-346 (2005).

[18] Smith, D. R., Padilla, W. J., Vier, D. C., NematNasser, S. C. \& Schultz, S. Composite medium with simultaneously negative permeability and permittivity. Phys.Rev. Lett. 84, 4184-4187 (2000).

[19] Pendry, J. B., Holden, A. J., Robbins, D. J. \& Stewart, W. J. Magnetism from conductors and enhanced non-linear phenomena. IEEE Trans. Microw. Theory Tech. 47, 2075-2084 (1999).

[20] Guzatov, D. V. \& Klimov, V. V. Spontaneous emission of chiral molecule placed near chiral sphere: full QED approach (in preparation).

[21] Li, X. \& Shapiro, M. Spatial separation of enantiomers by coherent optical means. J. Chem. Phys. 132, 041101 (2010).

\section{Acknowledgements}

We are grateful to D. Bloch for fruitful discussions of this topic. This work has been supported by the Russian Foundation for Basic Research (grants 11-02-91065, 1102-92002, 11-02-01272) and performed in the frame of International program of Scientific Cooperation ("P.I.C.S." No 5813) between C.N.R.S. and the Russian Foundation for Basic Research.

\section{Author contributions}

All authors have contributed to this paper and agree to its contents.

\section{Competing interests}

The authors declare that they have no competing financial interests.

\section{Correspondence}

Correspondence and requests for materials should be addressed to V.K. (email: vklim@sci.lebedev.ru). 